\title{
6 PROMOCIÓN DE LA INVESTIGACIÓN E IDENTIDAD DIGITAL: EL CASO DE LA UNIVERSIDADE DA CORUNA
}

\author{
Research promotion and digital identity: the case of the \\ Universidade da Coruña
}

\section{Viviana Fernández-Marcial y Llarina González-Solar}

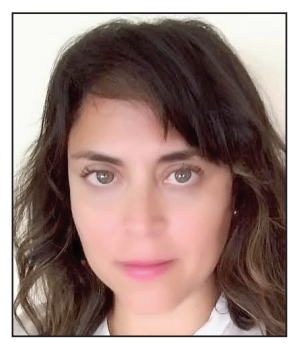

Viviana Fernández-Marcial es profesora titular del Área de Biblioteconomía y Documentación de la Universidade da Coruña (UDC). Doctora en documentación por la Universidad Carlos III de Madrid (2001), es profesora del Área de Biblioteconomía y Documentación desde 1997, año en que inicia su actividad docente en la Universidad San Pablo-CEU de Madrid. En 2006 se incorpora a la Facultade de Humanidades e Documentación de la UDC. Entre sus líneas fundamentales de investigación están el marketing de servicios de información y la comunicación científica. http://orcid.org/0000-0002-9277-266X

Universidade da Coruña, Facultad de Humanidades y Documentación Campus de Ferrol. 15471 Ferrol (A Coruña), España viviana.fernandez@udc.es

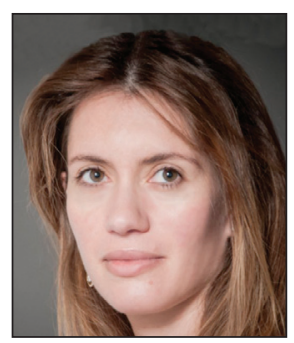

Llarina González-Solar es facultativo superior de bibliotecas en la Universidade da Coruña, en donde trabaja desde 2008, participando a lo largo de estos años en varios grupos de trabajo y en proyectos de traducción y de revisión de la CDU. Es licenciada en documentación (2004) y actualmente elabora su tesis doctoral en la Universidade da Coruña sobre los servicios a los investigadores en la biblioteca universitaria desde la perspectiva del marketing, línea de investigación ya comenzada en su trabajo fin de máster. http://orcid.org/0000-0003-4443-4102

Universidade da Coruña, Facultade de Ciencias, Biblioteca Campus Zapateira. 15008 A Coruña, España llarina.gonzalezs@udc.es

\section{Resumen}

Resultados de un estudio sobre la situación de la identidad digital en la comunidad investigadora de la Universidade da Coruña (UDC) Se toma como universo los docentes que figuran en el catálogo de investigación de la universidad y se examina su presencia en Orcid, ResearcherID, Scopus Author, Google Scholar Citations, ResearchGate y Mendeley. Los resultados reflejan que los niveles deben ser mejorados pero, en comparación con la bibliografía consultada, pueden valorarse como positivos. Se reflexiona sobre el papel que deben asumir los investigadores, la biblioteca universitaria y la institución.

\section{Palabras clave}

Comunicación científica; Identificadores; Redes sociales académicas; Redes sociales digitales científicas; Biblioteca universitaria; Identidad digital; Universidade da Coruña.

\begin{abstract}
The results of a study on the situation of digital identity of the research community of the University of A Coruña are presented. The universe has been established with the faculty members listed in the university's research catalog, and their presence in Orcid, ResearcherID, Scopus Author, Google Scholar Citations, ResearchGate and Mendeley was examined. The results show that the presence should be increased, but compared with others found in the literature, it can be assessed as positive. The role that researchers, the university library and the university must assume in this issue is suggested.
\end{abstract}

\section{Keywords}

Scientific communication; Identifiers; Social academic networks; Social scientific digital networks; Academic library; Digital identity; Universidade da Coruña. 
Fernández-Marcial, Viviana; González-Solar, Llarina (2015). “Promoción de la investigación e identidad digital: el caso de la Universidade da Coruña”. El profesional de la información, v. 24, n. 5, pp. 656-664.

http://dx.doi.org/10.3145/epi.2015.sep.14

\section{Introducción}

Los cambios en el sistema universitario provocados por el actual marco de ordenación, el Espacio Europeo de Educación Superior (EEES), y la estructuración de una nueva política en materia de investigación con la creación del Espacio Europeo de Investigación (EEI), han alterado el modo de concebir y gestionar la investigación universitaria. Las vías de financiación han quedado condicionadas por la rentabilidad y productividad científicas, y los indicadores de calidad se han convertido en factores clave para las universidades.

En nuestros días la excelencia universitaria se valora fundamentalmente por la posición, global o por áreas, que se ocupa en los rankings. Amsler y Bolsmann (2012) los definen como:

"un instrumento tangible de la política que en cierta medida redefine los propios fines sociales de la enseñanza superior".

Estos autores reflexionan sobre el hecho de que los rankings condicionan la financiación de la investigación y la docencia, crean una red de organizaciones de élite y constituyen un factor de exclusión social. Bourdieu et al. (1999, citado por Amsler y Bolsmann, 2012) sostienen que los rankings son:

"entendidos menos como indicadores de calidad y más como un producto de consumo".

El interés de las universidades por los rankings va más allá del prestigio académico e investigador que proporcionan y adquieren especial importancia las ventajas competitivas que éstos proporcionan (Gómez; Puente, 2013):

- obtener recursos y financiación de fondos públicos y privados;

- crear alianzas entre universidades y con otras entidades;

- atraer a un mayor número de estudiantes y a un profesorado de mayor prestigio.

A todo ello hay que añadir el impacto de los rankings en las estrategias de marketing de las universidades. Hazelkorn (2008) recoge los datos de un estudio internacional realizado en 2006, evidenciando que el $50 \%$ de las organizaciones de educación superior utilizan su posición en los rankings con fines promocionales.

Los principales rankings -Academic Ranking of World Universities, Times Higher Education, QS Top Universities- otorgan en sus cálculos un elevado peso a los resultados de la investigación. Así, las tareas orientadas a la promoción y mejora de la identidad y reputación digital redundan en la sostenibilidad, competitividad y estabilidad de las universidades mediante su presencia en rankings. Se asume que una investigación de calidad debe ser una investigación visible. Por ello, la identidad digital es una nueva dimensión de la calidad de la investigación.
Cabe definir la identidad digital como el resultado del esfuerzo consciente que realiza el investigador por y para ser identificado y reconocido en un contexto digital, distinguiéndose del conjunto de investigadores a través de la normalización, con el uso de identificadores, y la difusión de resultados de investigación en redes y plataformas de diversa naturaleza. Rotenberg y Kushmerick (2011) exponen que:

“los investigadores están bajo presión para encontrar colaboradores y mantenerse al día en las tendencias de su campo. La asociación y atribución apropiadas de los resultados de investigación son un imperativo para la gestión de la reputación y la identidad profesional. La identificación del nombre del autor y la correcta atribución de los resultados de investigación es crítico en la reputación y la carrera investigadora".

Lara (2009) aborda la noción de identidad digital desde la óptica de las instituciones al señalar que ésta:

"tiene que ver con la identificación que la propia institución hace de sí misma dentro de la cultura digital y con la postura que sea capaz de definir, apoyar, mantener y proyectar con respecto a su forma de relacionarse con las personas, con los contenidos y con las estructuras de producción y divulgación del conocimiento".

Además, precisa que identidad digital no se ciñe a la simple presencia en la Red a través de la creación de perfiles.

La identidad digital es el resultado del esfuerzo consciente que realiza el investigador por y para ser identificado y reconocido en un contexto digital

El concepto de reputación científica conecta con el de identidad digital. Puede ser definida como el prestigio de un investigador obtenido gracias a la calidad e impacto de sus resultados de investigación. Las tecnologías de la información están condicionando este concepto, así, es posible hablar de reputación digital, online o e-reputación, siendo su contrapartida la reputación offline. Necesariamente se plantea un estrecho vínculo entre ambos entornos de modo que, si el reconocimiento de un investigador se puede trasladar al contexto digital, una adecuada gestión de la identidad digital puede llevar a un mayor reconocimiento científico.

Los sistemas relacionados con la identidad digital actúan en dos ejes: la desambiguación y la visibilidad de los resultados de investigación.

En cuanto a la desambiguación Haak (2014) reivindica el interés de los identificadores persistentes para aclarar el papel de cada individuo en los flujos del trabajo científico, algo imprescindible dada la competencia para buscar fuentes de 
financiación. Su uso temprano en la carrera investigadora evitaría muchos malentendidos y ayudaría a que la información asociada a un investigador sea mucho más completa y precisa (Smith-Yoshimura et al., 2014). Estos sistemas posibilitan tanto una identificación consistente de los investigadores y la recogida de datos a un nivel más granular, como la agregación de tales datos generando agrupaciones en torno a un determinado investigador (como autor, colaborador...), una organización o una determinada fuente de financiación (Gunn, 2014; Carpenter, 2015).

Los sistemas de perfiles de investigadores actúan en dos ejes, la desambiguación y la visibilidad de los resultados de investigación, permitiendo conocer el historial científico de un investigador. Ayudan a la puesta en valor de la investiga- ción, al ser un medio para su difusión y dar soporte a indicadores altmétricos, todo lo cual revierte en un incremento de citas. Estas altmétricas se convierten en factor de identidad y reconocimiento (Taylor, 2012). Los investigadores utilizan estos sistemas fundamentalmente para comprobar si han sido contactados, encontrar nuevos colegas, comunicarse con ellos, compartir textos y acceder a las métricas (VanNoorden, 2014).

En un estudio de la OCLC, Smith-Yoshimura et al. (2014) proponen diez categorías de estos sistemas, incluyendo:

- plataformas de autoridades (authority hubs): proporcionan una ubicación centralizada para registros de autoridad de múltiples organizaciones;

- plataformas de identificadores (ID hubs): facilitan la crea-

Tabla 1. Descripción de sistemas relacionados con la identidad y la reputación científica digital

\begin{tabular}{|c|c|c|c|c|c|}
\hline Sistema & Creación & Tipo & Datos & Servicios & Tamaño \\
\hline Orcid & 2009 & $\begin{array}{l}\text { Plataforma de } \\
\text { identidades }\end{array}$ & $\begin{array}{l}\text { Número de identificación } \\
\text { Variantes de nombre } \\
\text { Filiación } \\
\text { Palabras clave } \\
\text { Datos curriculares y de financia- } \\
\text { ción } \\
\text { Vínculos con páginas web e } \\
\text { identificadores } \\
\text { Lista editable de publicaciones }\end{array}$ & $\begin{array}{l}\text { Cada información puede hacerse pública, } \\
\text { privada o visible a grupos concretos } \\
\text { Generación de códigos QR } \\
\text { APIs de apoyo a la comunicación y autenti- } \\
\text { cación de sistema a sistema } \\
\text { Sincronización con Scopus y ResearcherID }\end{array}$ & $\begin{array}{l}1.184 .753 \text { usuarios } \\
\text { (orcid.org febrero } \\
\text { 2015) }\end{array}$ \\
\hline ResearcherID & 2008 & $\begin{array}{l}\text { Plataforma de } \\
\text { autoridades }\end{array}$ & $\begin{array}{l}\text { Número de identificación } \\
\text { Variantes de nombre } \\
\text { Filiación } \\
\text { Palabras clave } \\
\text { Datos curriculares y de financia- } \\
\text { ción } \\
3 \text { listas editables de publicaciones } \\
\text { Indicadores bibliométricos }\end{array}$ & $\begin{array}{l}\text { Dos listas de publicaciones para realizar } \\
\text { análisis comparativos o rastrear los trabajos } \\
\text { de colegas } \\
\text { Creación de insignia (badge) } \\
\text { Búsqueda por palabras clave, nube de } \\
\text { etiquetas y geográfica } \\
\text { Controlar la privacidad de las informaciones } \\
\text { por partes } \\
\text { Integrado en las referencias de WoS Core } \\
\text { Collection }\end{array}$ & $\begin{array}{l}350.000 \text { miembros } \\
\text { (Smith-Yoshimura, } \\
\text { et al., 2014) }\end{array}$ \\
\hline $\begin{array}{l}\text { Scopus author } \\
\text { identifier }\end{array}$ & 2004 & $\begin{array}{l}\text { Identificador } \\
\text { dependiente de } \\
\text { la base de datos }\end{array}$ & $\begin{array}{l}\text { Número de identificación } \\
\text { Variantes de nombre } \\
\text { Filiación } \\
\text { Palabras clave } \\
\text { Lista no editable de publicacio- } \\
\text { nes } \\
\text { Indicadores bibliométricos }\end{array}$ & $\begin{array}{l}\text { Creación automática del Scopus author } \\
\text { profile } \\
\text { El autor puede corregir los fallos derivados } \\
\text { de algoritmos en la identificación } \\
\text { Visible gratuitamente en Free Scopus author } \\
\text { preview }\end{array}$ & \\
\hline $\begin{array}{l}\text { Perfil de Goo- } \\
\text { gle Scholar }\end{array}$ & 2011 & $\begin{array}{l}\text { Sistema de perfi- } \\
\text { les de investiga- } \\
\text { dores }\end{array}$ & $\begin{array}{l}\text { Nombre } \\
\text { Filiación } \\
\text { Palabras clave } \\
\text { Lista de referencias editable } \\
\text { Indicadores bibliométricos }\end{array}$ & $\begin{array}{l}\text { Creación voluntaria sobre la cuenta perso- } \\
\text { nal de Google pero requiere una dirección } \\
\text { de correo institucional para hacerse público } \\
\text { Localizable desde Google Scholar o desde } \\
\text { otro perfil } \\
\text { Crear alertas sobre las citas o documentos } \\
\text { asociados a un perfil }\end{array}$ & $\begin{array}{l}94.000 \text { perfiles } \\
\text { (Ortega; Aguillo, } \\
\text { 2014) }\end{array}$ \\
\hline ResearchGate & 2008 & $\begin{array}{l}\text { Sistema de perfi- } \\
\text { les de investiga- } \\
\text { dores }\end{array}$ & $\begin{array}{l}\text { Nombre } \\
\text { Filiación } \\
\text { Palabras clave } \\
\text { Descripción de proyectos } \\
\text { Habilidades (avaladas por otros } \\
\text { miembros) } \\
\text { Referencias y texto completo } \\
\text { Cálculo de altmétricas, } R G \text { Score }\end{array}$ & $\begin{array}{l}\text { Publicar el texto completo de los artículos } \\
\text { Foro de discusión Q\&A } \\
\text { Áreas privadas para el trabajo colaborativo, } \\
\text { Projects } \\
\text { Ofertas de trabajo } \\
\text { Solicitar y realizar revisiones, Open Review }\end{array}$ & $\begin{array}{l}\text { Más de } 5 \text { millones } \\
\text { de miembros } \\
\text { Más de } 67 \text { millones } \\
\text { de referencias (14 } \\
\text { a texto completo) } \\
\text { (ResearchGate.com } \\
\text { febrero de 2015) }\end{array}$ \\
\hline Mendeley & 2007 & $\begin{array}{l}\text { Sistema de } \\
\text { gestión de refe- } \\
\text { rencias / sistema } \\
\text { de perfiles de } \\
\text { investigadores }\end{array}$ & $\begin{array}{l}\text { Nombre } \\
\text { Filiación } \\
\text { Palabras clave } \\
\text { Datos curriculares y premios } \\
\text { Lista de publicaciones propias } \\
\text { Listas de publicaciones de interés }\end{array}$ & $\begin{array}{l}\text { Extraer automáticamente los metadatos de } \\
\text { documentos } \\
\text { Almacenamiento y anotación de documen- } \\
\text { tos en pdf } \\
\text { Elaboración de citas bibliográficas } \\
\text { Genera estadísticas por disciplina, país o } \\
\text { nivel académico } \\
\text { Formar grupos de investigación con carpe- } \\
\text { tas compartidas } \\
\text { Búsqueda de personas con intereses simila- } \\
\text { res y seguimiento }\end{array}$ & $\begin{array}{l}\text { 3,1 millones de } \\
\text { miembros (Van- } \\
\text { Noorden, 2014) }\end{array}$ \\
\hline
\end{tabular}


ción de un registro centralizado de identificaciones;

- sistemas de gestión de referencias (reference management systems): ayudan a los investigadores organizar el trabajo, colaborar y descubrir nuevas publicaciones;

- sistemas de perfiles de investigadores (researcher profile systems): posibilitan la creación de redes profesionales.

Algunos de los sistemas más conocidos son brevemente descritos en la tabla 1.

\section{La investigación en la UDC: perfil de los investigadores y producción científica}

La investigación en la Universidade da Coruña (UDC) se gestiona a través de diferentes servicios, que proporcionan soporte a los investigadores y que incluyen:

- Oficina de Transferencia de Resultados de Investigación (OTRI);

- Servicio de Publicaciones;

- Oficina de Software Libre;

- Centro Universitario de Formación e Innovación Educativa (Cufie);

- Servicios de Apoyo a la Investigación (SAl);

- Servicio de Investigación (Serin) encargado de la gestión administrativa del I+D+i;

- Biblioteca que cuenta con una sede central y 17 puntos de servicio.

La UDC no figura en las principales clasificaciones internacionales y su posición no es alta en las nacionales, tal como puede apreciarse en el documento Situación da UDC nos rankings internacionales (UDC, s.f.). Sin embargo, tomando como base SCImago institutions rankings (SIR), se evidencia una mejora más o menos constante, en indicadores como:

- colaboración internacional;

- número total de publicaciones recogidas por Scopus;

- número de investigadores diferentes que publican en revistas recogidas por Scopus;

- ratio de publicaciones en el primer cuartil.

De acuerdo con los datos de la memoria de la UDC para el curso 2014/2015, la universidad cuenta con un total de 1.416 profesores, distribuidos en 43 departamentos y ubicados en 25 escuelas y facultades. Para este trabajo se realiza una primera valoración de la comunidad investigadora tomando como referencia el Catálogo de investigación de la UDC. Se asume que el Catálogo recoge la casi totalidad de los investigadores en activo de la universidad pues para formar parte de él es necesario contar con resultados de investigación en los últimos cinco años. No obstante, dado que el alta y actualización anual de los datos compete al propio investigador, es preciso asumir que existirá un bajo porcentaje de docentes que no figuren en el catálogo, aún cumpliendo los requisitos.

El Catálogo de investigación se compone de tres tipos de entidades:

- grupo de investigación;

- unidad de investigación;

- investigador individual.

La normativa establece los criterios que regulan la composición y requerimientos para cada uno de ellos.
En el caso del investigador individual debe ser verificable una producción científica en los últimos cinco años y es necesario ser personal docente e investigador (PDI) doctor.

Tanto la unidad como el grupo son entidades formadas por múltiples investigadores y lideradas por un investigador principal o coordinador. En el caso de la unidad de investigación este coordinador debe cumplir las mismas condiciones que los investigadores individuales. El coordinador de un grupo de investigación debe poseer los mismos requisitos y, además, contar con cierta antigüedad como doctor, una financiación continuada como investigador principal, sexenios de investigación activos y tesis doctorales dirigidas. Cada miembro de la UDC sólo puede figurar en un grupo, en una unidad o como investigador individual, de modo que no puede figurar en dos de estas categorías.

Los tres ejes sobre los que articula el trabajo en torno a la reputación e identidad digital son, el investigador, la biblioteca universitaria y la propia universidad

Durante la elaboración del trabajo no se encontraban disponibles las estadísticas realizadas por la OTRI para 2014 sobre la composición del catálogo. Se revisaron los datos de 2013, para tener una primera valoración del universo y poder contrastar con los actuales. Los datos más recientes se obtuvieron mediante la consulta directa al catálogo, actualizado en diciembre de 2014. Se realizó un vaciado de los datos y se elaboraron tablas en Excel con la denominación de:

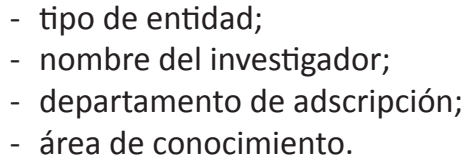

Debido a las limitaciones de interrogación del Catálogo se optó por realizar el estudio sobre las entidades y no sobre el total de investigadores. Esto implica que en el caso de grupos y unidades de investigación se analizase únicamente el investigador principal. El universo de la investigación está constituido por el total de entidades.

La comparación de datos del curso anterior y el actual muestra un ligero descenso del total de entidades. Si para el curso 2013/2014 el número total era de 252, en el siguiente existían 232. Esta caída se produce en el número de investigadores individuales (de 96 a 84), pero también ocurre en los grupos (de 126 a 119) y en tan sólo un punto en las unidades de investigación (de 30 a 29). Una valoración global de estas cifras apunta a la colaboración como un rasgo distintivo de la investigación en la UDC. Los datos de ambos cursos muestran que más de la mitad de la investigación se localiza en unidades y grupos de investigación y que hay una tendencia de crecimiento al pasar de $61,9 \%$ a $63,8 \%$.

En la figura 1 se aprecian los datos de distribución de la investigación y permite realizar algunas observaciones:

- El mayor porcentaje de investigadores se localiza en el área de ciencias sociales y jurídicas con 84 entidades, seguido por la de ingeniería y arquitectura (69 entidades). 
Un menor porcentaje aparece en artes y humanidades (31), ciencias (28) y ciencias de la salud (20). Para cualificar estos resultados es preciso valorarlos en el contexto de la UDC. Las dos primeras son las áreas emblemáticas de la universidad y las que aglutinan un mayor número de profesorado. Así, para el curso 2012/2013 ciencias sociales y jurídicas tenía 441 docentes y las enseñanzas técnicas un total de 501, mientras que ciencias de la salud tenía 134 y humanidades 189.

- La colaboración científica, a través de grupos o de unidades de investigación (148 en total) es alta en las áreas de ingeniería y arquitectura, que representa el 35\% del total de equipos de la UDC, de ciencias sociales y jurídicas $(30,4 \%)$ y ciencias $(23 \%)$. Si se observa la distribución dentro de cada área, en ingeniería y arquitectura el trabajo en equipos ocupa el $75,4 \%$ de toda su investigación pero igualmente significativo es en el área de ciencias donde este porcentaje asciende a $82,1 \%$.

- Si se toma el grupo de investigación como criterio de excelencia, en función de los parámetros establecidos en la normativa, es posible apuntar que se concentra en las tres áreas donde este número es mayor y que coincide con las citadas en el punto anterior.

- En el caso de los investigadores individuales, las áreas que concentran un mayor número en relación con el resto son por este orden: ciencias sociales y jurídicas, ingeniería y arquitectura $y$, artes y humanidades. En ninguna de las áreas el número de investigadores individuales supera al de grupos y unidades, confirmando la tendencia expuesta en el primer punto. No obstante, existe una distribución interna sui generis en las áreas de ciencias sociales y jurídicas y de artes y humanidades, pues los porcentajes entre equipos e investigadores individuales son relativamente equitativos.

Algunos datos de producción científica de la UDC muestran resultados estables con cierta tendencia a su incremento en los cuatro últimos años. El total de artículos recogidos en WoS entre 2011-2014 llega a 1.945 registros, con una media aproximada de 500 incorporaciones anuales. En Scopus las cifras son similares aunque ligeramente superiores con 2.027 artículos. Las actas de congreso llegan a 188 y 599 respectivamente en idéntico período. La UDC ha destacado en la actividad de patentes y registro de la propiedad intelectual. Entre 2009 y 2012 se han concedido 27 patentes españolas y se han efectuado 45 registros de la propiedad intelectual, incluidos softwares (BUDC, 2015). Los resultados de la actividad científica para 2013 y 2014 muestran igualmente una tendencia positiva en algunos capítulos, tal como puede observarse en los indicadores publicados en el

Portal da transparencia de la UDC. https://www.udc.gal/transparencia/resultados

\section{Identidad digital de los investigadores de la UDC}

La investigación sobre la identidad digital de los investigadores de la universidad implicó necesariamente una limitación en cuanto a la muestra y a los sistemas. Se optó por observar el total de entidades presentes en el Catálogo de investigación pero se determinó que, en el caso de los grupos y unidades de investigación, el análisis se centraría en el investigador principal y no en el total de los miembros. En una fase previa se habían identificado los investigadores por departamento, centro y área de conocimiento. Se analizaron los 232 investigadores. Se definieron como medios más representativos aquellos que son referenciados con mayor frecuencia en la literatura científica, entre ellos los trabajos de Van-Noorden (2014) y Smith-Yoshimura et al. (2014). Así, se analizaron las caracterizadas en el apartado 3: Orcid, ResearcherID, perfil de Google Scholar, Scopus, ResearchGate y Mendeley.

Para Orcid se han estudiado los perfiles claramente identificables y su calidad, valorando la vinculación con otros sistemas y la inclusión de datos curriculares y de publicaciones. Se descartaron los perfiles con datos básicos, al no tener la certeza de que correspondan al investigador.

En Scopus Author fueron anotados los perfiles únicos, duplicados, autores sin presencia o reducida a una única publicación. Se han tenido en cuenta los Profile matches with one document, que suponen la principal causa de duplicidad. Se detectó un bajo número de perfiles que aglutinaban datos de varios investigadores, lo cual no ha afectado a los cálculos si bien se ha tomado nota de ello.

En ResearcherID se contabilizaron los perfiles identificables y la fecha de su última actualización. En el análisis del perfil de Google, ResearchGate y Mendeley únicamente se valoró la presencia en los mismos. En todos los casos se tuvieron en cuenta las variantes posibles de los nombres y en algunos casos se completó la búsqueda con la filiación, que también se utilizó como filtro. Los resultados generales del 
trabajo pueden apreciarse en la figura 2. Se observa que $R e$ searchGate es el sistema de creación activa de perfiles más utilizado.

Un $21 \%$ de los investigadores tienen perfil en Orcid, correspondiendo el porcentaje más alto a los de ciencias (43\%) y el más bajo a los de humanidades (10\%). Estos datos están muy por encima de los obtenidos a nivel internacional por Van-Noorden (2014) que indica un escaso $10 \%$ en ciencias e ingeniería y un dato inferior para ciencias sociales, artes y humanidades. La mayoría de los perfiles son bastante completos, con enlaces a otras plataformas y/o información curricular detallada. En concreto, los investigadores de ciencias llegan a vincular en un $92 \%$ de los casos sus perfiles a Web of Science y Scopus, donde también tienen una mayor presencia.

\section{Los criterios de selección de un medio social u otro depende de que haya una masa crítica de colegas}

El nivel de uso del perfil de Google y de ResearcherID es similar, $14-16 \%$ respectivamente; el primero es más frecuente entre los investigadores de ingeniería y arquitectura, puntualmente del área de informática (47\%) y el segundo entre los de ciencias (39\%). El porcentaje de perfiles de $R e$ searcherID creados o actualizados en 2014-2015 es elevado, un $63 \%$ del total, siendo un $100 \%$ de investigadores de ciencias de la salud. Esto podría indicar que estos investigadores han recibido información recientemente sobre el sistema, por ejemplo para participar en convocatorias de proyectos competitivos.

El 73\% de los investigadores analizados aparece en Scopus. Este dato no es comparable con los anteriores ya que la voluntad o actuación del investigador no resulta determinante para su presencia en la base de datos. Esta depende de que Scopus recoja su producción científica. La faceta activa del investigador consiste en el trabajo de desambiguación de su perfil. El número de autores con un único perfil es elevado (40\%) pero desciende al $28 \%$ si sólo se consideran aquellos con más de una publicación asociada.

ResearchGate es la plataforma de creación de perfiles más utilizada por los investigadores de la UDC, con un 39\% de los casos observados. Estos datos son elevados si se comparan con el estudio de Nature (Van-Noorden, 2014) que lo sitúa como el primero de los sistemas estudiados y observa un uso del $35-45 \%$.

Para Gunn (2013), Mendeley es una red social académica de gran aceptación en las áreas de ciencias de la vida, química, matemáticas, ciencias de la computación y con cierto grado de acogida en las ciencias sociales. Sin embargo en este trabajo se evidencia que su utilización es la más baja (8\%) siendo consistente con los datos obtenidos por VanNoorden (2014).

Se ha detectado un pequeño grupo de investigadores que mantienen una presencia constante en casi todos los medios, Orcid, ResearcherID, Google, ResearchGate y un Scopus Author único. Los mismos pertenecen a áreas diversas:

- informática (3)

- biología (3)

- psicología (2)

- podología (1)

- educación física (1)

- ingeniería (1).

Esta evidencia la podemos considerar como un interés de estos docentes por trabajar su identidad digital.

Las grandes áreas han demostrado ser heterogéneas, especialmente la agrupación de informática, ingeniería y arquitectura. Las dos primeras muestran datos similares para varios puntos (en torno al $30 \%$ en Orcid y $25 \%$ en ResearcherID) y discrepancias en otros ( $47 \%$ y $14 \%$ en Google y $59 \%$ y $51 \%$ respectivamente en ResearchGate). Estos datos contrastan con la escasa presencia de arquitectura, presumiblemente a causa de peculiaridades de la comunicación científica y, en cierta medida, el carácter más local de su investigación.

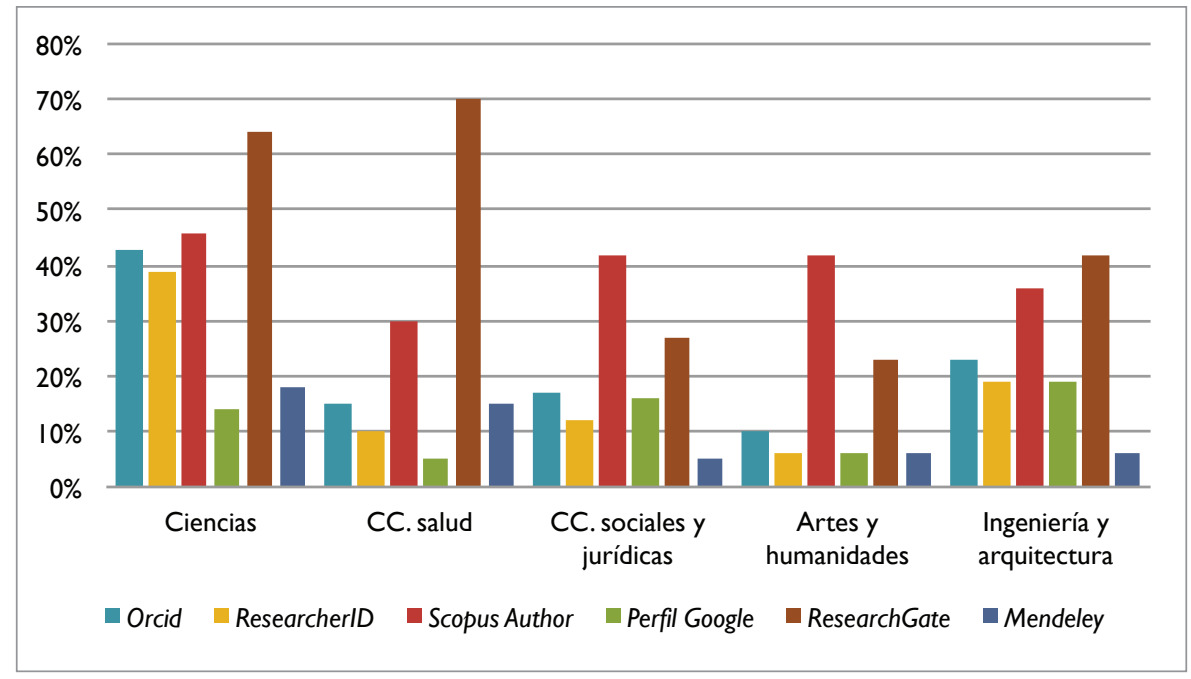

Figura 2. Presencia porcentual por áreas
Se puede comprobar que no existe una correlación entre el número de investigadores en un área y su presencia en los sistemas de identidad digital. Lo demuestran las áreas de ciencias y ciencias de la salud, que ocupan la primera y segunda posición con relación a presencia en los sistemas de identidad digital estudiados; y son ambos casos y por idéntico orden las que menor número de investigadores tienen.

\section{Conclusiones}

Los resultados del trabajo nos ofrecen la imagen global de los investigadores de la UDC en cuanto a la gestión de su identidad digital. La comparación con los obtenidos por 


\section{Ciencias e ingeniería}

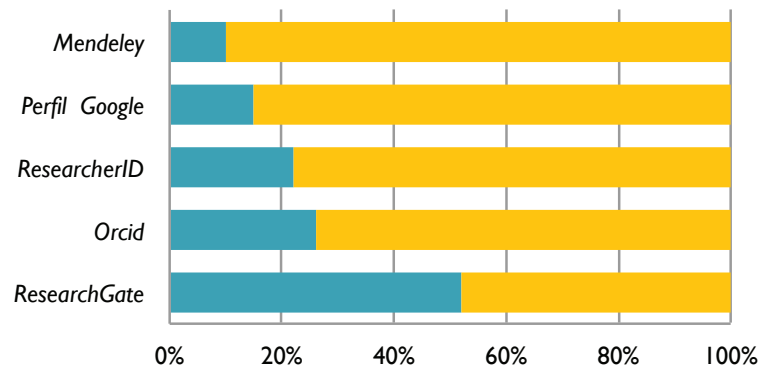

\section{Ciencias sociales y humanidades}

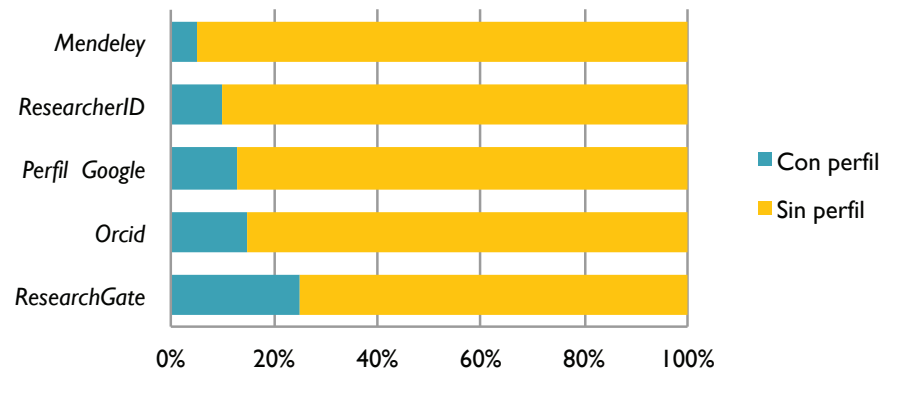

Figura 3. Distribución de sistemas agrupados por bloques

Van-Noorden (2014) indica una orientación similar tanto en preferencias (por ejemplo el mayor uso de ResearchGate) como en niveles de uso. Los datos para la UDC en cuestiones clave como el porcentaje de perfiles Orcid son mejores que los presentados en el citado estudio y, sin embargo, aún queda un largo camino por recorrer.

Los tres ejes sobre los que articula el trabajo en torno a la reputación e identidad digital son: el investigador, la biblioteca universitaria y la propia universidad.

Los medios que promueven la comunicación científica informal adquieren más relevancia

\section{Investigadores}

El avance en esta dirección estará condicionado por las reglas que dominan la comunicación científica:

- La presión que ejerce una determinada comunidad o área científica sobre sus miembros puede ser decisiva para tener presencia en medios. Se puede producir un efecto llamada y constituir un elemento de cohesión. Los criterios de selección de un sistema u otro parece que van depender de que haya una masa crítica de colegas y están influidos por imperativos nacionales o de búsqueda de financiación (Smith-Yoshimura et al., 2014)

- El índice de participación puede ser inferior en áreas científicas en las que la transmisión de conocimiento se realiza a través de monografías y/o publicaciones científicas no sistematizadas en las grandes bases de datos, algo característico en artes y humanidades y en determinados sectores de las ciencias sociales.

- Los investigadores están siendo impulsados a crear perfiles por agentes externos, editoriales, agencias de evaluación, revistas científicas... Ello produce una cierta desviación de la naturaleza de estos sistemas que requieren un papel activo y sistemático del investigador. Puede darse el extremo de que un investigador para publicar una única vez en una revista cree uno o más perfiles que luego queden desactualizados.

- Estos medios tienen un carácter promocional y ofrecen una visibilidad que no siempre estará respaldada por una producción alta o media. De hecho, su uso puede resultar muy atractivo para investigadores con escasa producción científica, e investigadores con trayectoria científica consolidada pueden no tener interés en esta faceta promocional ni en vincularse con redes o medios que amplíen su notoriedad y contactos, pues ya cuentan con ello.

- Aquellos medios que promueven la comunicación científica informal adquieren relevancia. Pese a que ResearchGate no está relacionada con los indicadores bibliométricos tradicionales tiene una posición ventajosa, puede que debida a su carácter social que conecta con el cambio de paradigma en la comunicación científica.

- Se percibe un nivel de uso dispar entre los medios y se observa una escasa relación entre las exigencias de calidad científica y el uso de estos sistemas. Así, el número de perfiles en ResearcherID es bajo pese a la importancia de los JCR en la carrera investigadora. En consonancia con los datos de Van-Noorden (2014) se evidencia, que pocos investigadores conocen Orcid y menos aún lo utilizan. Otro tanto ocurre con Mendeley.

- Existe un alto número de investigadores sin presencia en ninguno de los sistemas y son escasos los que aparecen en todos. Esto sugiere que, en general, existe un cierto desconocimiento de estos sistemas.

\section{Biblioteca}

Es necesario señalar su papel activo y crucial en este proceso de construcción de la identidad y la reputación digital de los investigadores, pero también los límites de su participación. Diversos autores han abordado este problema poniendo el énfasis en una responsabilidad compartida entre la biblioteca y el investigador.

Con relación a Orcid, De-Castro (2015) expresa que la intervención institucional en la carga masiva de los datos puede generar mayores dificultades que soluciones y que la biblioteca debería asumir una función didáctica, más que sustituir al investigador en su tarea. También Alonso-Arévalo (2014) ve la alfabetización científica como el camino para intervenir en la identidad y reputación de los investigadores ya que esta "puede ser potenciada y gestionada" para lo que "el investigador puede encontrar un aliado en el propio bibliotecario".

El informe de la OCLC (Smith-Yoshimura et al., 2014) otorga un papel más activo a la biblioteca recomendando la realización de trabajos como la asignación de identificadores a 
los autores que carezcan de ellos, integración de estos identificadores con las aplicaciones y servicios internos, además de educar en el uso y los beneficios de estos sistemas. Igualmente define las tareas que deben asumir, investigadores, gestores de la universidad y editores.

Rotenberg y Kushmerick (2011) inciden en la necesidad de solucionar la cuestión de la identidad e identificación de autores a través de la combinación de trabajo manual y automatizado, con la intervención institucional e individual de los investigadores. La biblioteca, en su papel mediador, puede desencadenar los procesos de identidad y reputación digital que deben ser continuados por el investigador. En otras palabras, la biblioteca puede crear un perfil en Research Gate pero no suplantar la intervención del investigador en los foros o en el intercambio de información, ni tiene capacidad para actualizar la información de toda una comunidad. Las líneas de actuación de la biblioteca de la UDC para trabajar en la gestión de la identidad digital de los investigadores pueden ser varias:

La identidad y reputación digital debe contar con una estrategia institucional coordinada de todos los servicios y con un marketing interno

- creación de un servicio para la investigación, no como una simple reorganización de recursos de información de apoyo;

- en el marco de esta estructura organizacional, se debería integrar la gestión de la identidad digital como una de sus funciones;

- programa de formación y asesoramiento a los investigadores sobre los medios y sistemas disponibles para la gestión de la identidad digital así como el diseño de un microsite con toda la información y recursos disponibles;

- actuar a través de los departamentos y/o grupos de investigación a modo de bibliotecarios integrados.

La biblioteca puede emprender de forma autónoma actividades como la correcta descripción de las filiaciones de investigadores de la UDC en base de datos relevantes, por ejemplo Web of Science, reasignando estos documentos. También la integración de los sistemas de identidad digital con los propios de la biblioteca, por ejemplo, la identificación de los autores en el repositorio a través de Orcid. Todas estas actividades necesariamente deberán ir acompañadas de un plan de promoción continuado, no de acciones aisladas. Este plan debe tener como objetivo concienciar y aumentar la participación de los investigadores en la gestión de su identidad digital.

\section{Universidad}

La presencia de sus investigadores en los sistemas de identidad digital, refuerza y consolida su imagen y prestigio. La cuestión central está en que la identidad y la reputación digital deben formar parte de una estrategia institucional. La $U D C$ ha iniciado actuaciones para intensificar la gestión de la identidad digital entre sus investigadores. Así, la aplicación para la gestión curricular del personal investigador SUXI
(Sistema Universitario de Xestión da Investigación) integra actualmente los números de identificación de Orcid, ResearcherID y Scopus Author. Es posible considerar esta actividad como un avance pero un verdadero planteamiento de una estrategia institucional debe evolucionar hacia la adopción de medidas orgánicas y estratégicas que articulen y coordinen todos los servicios de apoyo a la investigación y, a la vez, se ponga en marcha un plan de marketing interno con vistas a sensibilizar a la comunidad investigadora sobre la importancia de la gestión de su identidad y reputación digital.

\section{Bibliografía}

Alonso-Arévalo, Julio (2014). "Alfabetización en comunicación científica: acreditación, OA, redes sociales, altmetrics, bibliotecarios incrustados y gestión de la identidad digital" En: Alfabetización informacional: reflexiones y experiencias, Lima (Perú), 20 y 21 de marzo.

http://hdl.handle.net/10760/22838

Amsler, Sarah S.; Bolsmann, Chris (2012). "University ranking as social exclusion". British journal of sociology of education, v. 33, n. 2, pp. 283-301.

http://dx.doi.org/10.1080/01425692.2011.649835

BUDC (2015). "O output da actividade científica da UDC". BUDC.información: blog da sección de Información e Coordinación da Biblioteca da Universidade da Coruña, 25 de febrero.

http://budcinformacion.blogspot.com.es/2015/02/ooutput-da-actividade-cientifica-da-udc.html

Carpenter, Todd (2015). "Why assessment needs persistent identifiers like Orcid". Orcid Blog, 27 de enero.

http://orcid.org/blog/2015/01/27/why-assessment-needspersistent-identifiers-orcid

De-Castro, Pablo (2015). "Implantación institucional del identificador orcid: un nuevo rol para las bibliotecas universitarias". Anuario ThinkEPI, v. 9, pp. 132-134.

http://dx.doi.org/10.3145/thinkepi.2015.31

Gómez, Tomás; Puente, Xavier (2013). "Rankings y reputación universitaria: Jornadas de la Augac, Universidad de La Rioja (segunda parte)".

http://asus.usal.es/tomas-gomez-y-xavier-puente/22317-alias

Gunn, William (2013). "Social signals reflect academic impact: what it means when a scholar adds a paper to Mendeley". Information standards quarterly, v. 25, n. 2, pp. 33-39. http://www.niso.org/apps/group_public/download.php/11275/ IP_Gunn_Mendeley_isqv25no2.pdf

http://dx.doi.org/10.3789/isqv25no2.2013.06

Gunn, William (2014). "On numbers and freedom". El profesional de la información, v. 23, n. 5, pp. 463-466. http://dx.doi.org/10.3145/epi.2014.sep.02

Haak, Laurel L. (2014). “Persistent identifiers can improve provenance and attribution and encourage sharing of research results". Information services and use, v. 34, n. 1-2, pp. 93-96.

http://dx.doi.org/10.3233/ISU-140736

Hazelkorn, Ellen (2008). "Globalization, internationalization, and rankings". International higher education, interna- 
tionalization, pp. 8-11.

http://arrow.dit.ie/cgi/viewcontent.cgi?article=1042\&conte $x t=c s e r a r t$

Lara, Tíscar (2009). "El papel de la universidad en la construcción de su identidad digital". Revista de universidad y sociedad del conocimiento, v. 6, n. 1, pp. 15-21.

http://dx.doi.org/10.7238/rusc.v6i1.25

Ortega, José-Luis; Aguillo, Isidro F. (2014). “Microsoft academic search and Google scholar citations: comparative analysis of author profiles". Journal of the Association for Information Science and Technology, v. 65, n. 6, pp. 11491156.

http://dx.doi.org/10.1002/asi.23036

Rotenberg, Ellen; Kushmerick, Ann (2011). "The author challenge: identification of self in the scholarly literature". Cataloging and classification quarterly, v. 49, n. 6, pp. 503-520. http://dx.doi.org/10.1080/01639374.2011.606405

Smith-Yoshimura, Karen; Altman, Micah; Conlon, Michael; Cristán, Ana-Lupe; Dawson, Laura; Dunham, Joanne; Hick- ey, Thom; Hook, Daniel; Horstmann, Wolfram; MacEwan, Andrew; Schreur, Philip; Smart, Laura; Wacker, Melanie; Woutersen, Saskia (2014). Registering researchers in authority files. Dublin, Ohio: OCLC Research. ISBN: 9781 556534881

http://www.oclc.org/content/dam/research/publications/ library/2014/oclcresearch-registering-researchers-2014.pdf

Taylor, Michael (2012). "The new scholarly universe: are we there yet?". Insights: the UKSG journal, v. 25, n. 1, pp. 12-17. http://dx.doi.org/10.1629/2048-7754.25.1.12

UDC (2014). Catálogo de investigación de la Universidade da Coruña.

http://investigacion.udc.es/es

UDC (s. f.). Portal da Transparencia da UDC: Resultados. https://www.udc.gal/transparencia/resultados

Van-Noorden, Richard (2014). “Online collaboration: scientists and the social network". Nature, v. 512, n. 7513, pp. 126-129.

http://dx.doi.org/10.1038/512126a

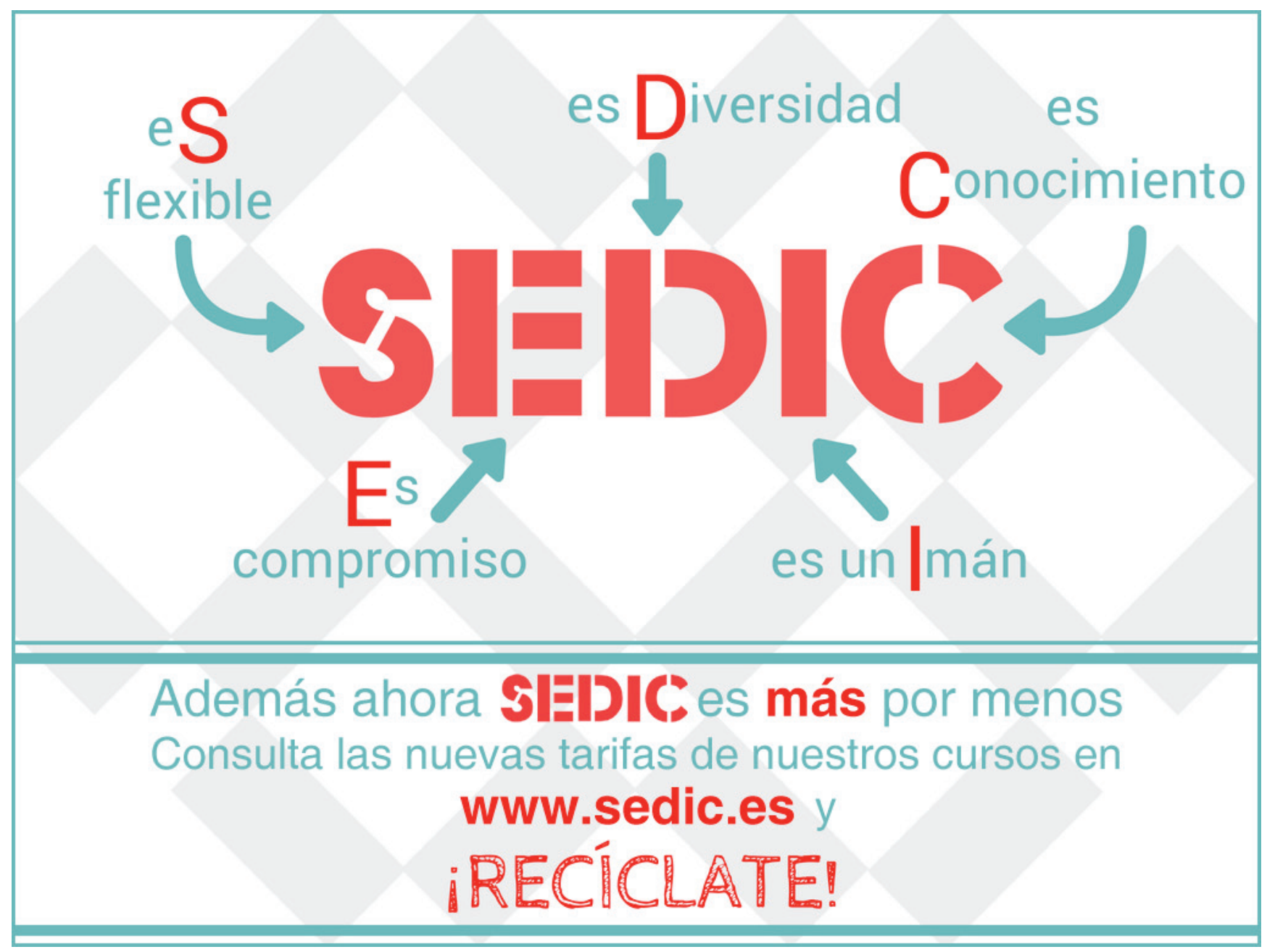

